\title{
Vector Median M-Type L Filter to Process Multichannel Images
}

\author{
Antonio Toledo-Lopez, Francisco J. Gallegos-Funes, and Volodymyr Ponomaryov \\ National Polytechnic Institute of Mexico \\ Mechanical and Electrical Engineering Higher School \\ UPALM Zacatenco, Av. IPN s/n, Edificio Z, Acceso 3, 3er. piso; \\ Col. Lindavista, 07738, Mexico D.F., Mexico \\ Phone/Fax: +52-55-57296000x54622 \\ fgallegosf@ipn.mx
}

\begin{abstract}
The Vector Median M-type L (VMML) -filter to remove impulsive noise from color images and video color sequences is presented. This filter utilizes multichannel image processing by using the vector approach and the Median M-Type L (MML) algorithm. Simulation results indicate that the proposed filter consistently outperforms other color image filters by balancing the tradeoff between noise suppression, detail preservation, and color retention.
\end{abstract}

Keywords: Median M-Type L algorithm, Multichannel image processing, Impulsive noise suppression.

\section{Introduction}

Many useful techniques of multichannel signal processing based on vector processing have been investigated due to the inherent correlation that exists between the image channels compared to traditional component-wise approaches [1,2]. Many applications of this technique are color image processing, remote sensing, robot vision, biomedical image processing, and high-definition television (HDTV) [1]. Different filtering techniques have been proposed for color imaging. Particularly, nonlinear filters applied to color images have been designed to preserve edges and details, and remove impulsive noise [1-3].

In this paper, we introduce the Vector Median M-Type L (VMML) -filter. This filter utilizes multichannel image processing by using the vector approach [2,4], and the Median M-Type L (MML) algorithm [5,6]. The proposed filter uses the combined RM-estimator [7] into the L-filter [8] by following way. The redescending $M$-estimator with different influence functions is combined with the $R$ - (median) estimator into the $L$ filtering scheme to obtain sufficient impulsive noise suppression for each channel by using the vector approach. We also introduce the use of an impulsive noise detector [9] to improve the properties of noise suppression and detail preservation in the proposed filtering scheme.

Simulation results have demonstrated that the proposed VMML filter consistently outperforms other color image filters by balancing the tradeoff between noise suppression, detail preservation, and color retention. 


\section{Proposed Multichannel MM Filters}

The proposed VMML filter employs the $L$ algorithm [8]. The following representation of $L$ filter is often used,

$$
\theta_{\mathrm{L}}=\sum_{k=1}^{N} a_{k} \cdot X_{(k)}
$$

where $X_{(k)}$ is the ordered data sample, $a_{k}=\int_{k-1 / N}^{k / N} h(\lambda) d \lambda / \int_{0}^{1} h(\lambda) d \lambda$ are the weighted coefficients, and $h(\lambda)$ is a probability density function [8].

For convenience the VL (Vector L) filter is written below as

$$
\theta_{\mathrm{L}}=\sum_{m=1}^{N} a_{m} \cdot \psi\left(y_{m}\right) y_{m}
$$

where $y_{m}$ are the noisy image vectors in sliding filter window, which includes $m=1, \ldots, N$ vectors $y_{1}, y_{2}, \ldots, y_{N}$ located at spatial coordinates in the filter window, and $\psi\left(y_{m}\right)=\left\{\begin{array}{ll}1 & m \leq(2 L+1)^{2} \\ 0 & \text { otherwise }\end{array}\right.$ is the influence function.

To improve the robustness of the VL filter, we propose to use the Median M-type estimator [5-7],

$$
\theta_{\mathrm{MM}}=\operatorname{MED}\left\{Y_{k} \widetilde{\psi}\left(Y_{k}-\operatorname{MED}\left\{Y_{N}\right\}\right), k=1, \ldots, N\right\}
$$

where $Y_{k}$ is data sample, $\widetilde{\psi}$ is the normalized influence function $\psi$ : $\psi(Y)=Y \tilde{\psi}(Y)$, and $Y_{N}$ is the primary data sample. The Median estimator provides good properties of impulsive noise suppression and the M-estimator uses different influence functions to provide better robustness, for these reasons it can be expected that the properties of combined MM-estimator could be better in comparison with Median and M- estimators [7].

So, the Vector Median M-type L (VMML) -filter can be written as:

$$
\theta_{\mathrm{VMML}}=\operatorname{MED}\left\{a_{m} \cdot\left[Y_{m} \psi\left(Y_{m}-\operatorname{MED}\left\{Y_{N}\right\}\right)\right]\right\} / a_{\mathrm{MED}}
$$

where $Y_{m} \psi\left(Y_{m}-\operatorname{MED}\left\{Y_{N}\right\}\right)$ is set of values of vectors $y_{m}$ which are weighted by value in accordance with the influence function $\tilde{\psi}\left(y_{m}\right)$ in a sliding filter window, $y_{m}$ are the noisy image vectors in a sliding filter window, which includes vectors $y_{1}, y_{2}, \ldots, y_{N}$ located at spatial coordinates $(i, j)$ in the filter window, $a_{m}$ are the weighted coefficients used into the proposed filtering scheme, and $a_{\mathrm{MED}}$ is the median of coefficients $a_{m}$ used as a scale constant. The simple cut and Andrew's sine influence functions are used in the filtering scheme [7], 


$$
\begin{gathered}
\psi_{\operatorname{cut}(r)}\left(y_{m}\right)=y_{m} \cdot 1_{[-r, r]}\left(y_{m}\right)= \begin{cases}y_{m}, & \left|y_{m}\right| \leq r \\
0, & \text { otherwise }\end{cases} \\
\psi_{\sin (r)}\left(y_{m}\right)= \begin{cases}\sin \left(y_{m} / r\right), & \left|y_{m}\right| \leq r \pi \\
0, & \text { otherwise }\end{cases}
\end{gathered}
$$

where the parameter $r$ is connected with restrictions on the range of $\psi\left(y_{m}\right)$.

We also propose to enhance the removal ability of VMML filter to involve an impulsive noise detector. The impulsive detector used here is defined as [9]:

$$
\left[\left(\operatorname{rank}\left(y_{c}\right) \leq T_{1}\right) \vee\left(\operatorname{rank}\left(y_{c}\right) \geq N-T_{1}\right)\right] \wedge\left(\left|y_{c}-\operatorname{MED}\left(Y_{N}\right)\right| \geq T_{2}\right)
$$

where $y_{c}$ is the vector of interest (the central vector in the filtering window), $T_{1}>0$ and $T_{2} \geq 0$ are thresholds, and $N$ is the length of the data.

The parameters for VMML filter were found after numerous simulations in different test images degraded by impulsive noise. The idea was to find the parameters values when the values of criteria PSNR and MAE would be optimum. In our experiments, a 3x3 sliding window was applied. The parameter of the Andrew's sine influence function is $r \leq 31$ and for the impulsive noise detector $T_{1}=3$ and $T_{2}=0.3 \cdot \operatorname{med}\left\{Y_{N}\right\}$. To compute the weighted coefficients we use the exponential, Laplacian and uniform distribution functions.

\section{Simulation Results}

The described VMML filter with the simple cut (S) and Andrew's sine (A) influence functions, the exponential (E), Laplacian (L), and uniform (U) distribution functions and, the impulsive noise detector (D) and without it (ND) has been evaluated, and its performance has been compared with vector median (VM), $\alpha$-trimmed mean $(\alpha$-TM), basic vector directional (BVD), generalized vector directional (GVD), adaptive GVD (AGVD), double window GVD (GVD_DW), multiple non-parametric (MAMNFE), vector median M-type K-nearest neighbor (VMMKNN), and fast adaptive similarity VM (FASVM) filters [4,10,11,12].

The criteria used to compare the restoration performance of various filters were the peak signal-to-noise ratio (PSNR) for evaluation of noise suppression, the mean absolute error (MAE) for quantification of edges and detail preservation, the mean chromaticity error (MCRE) for evaluation of chromaticity retention, and the normalized color difference (NCD) for quantification of color perceptual error [1-4]:

$$
\begin{gathered}
\mathrm{PSNR}=10 \cdot \log \left[(255)^{2} / \mathrm{MSE}\right], \mathrm{dB} \\
\mathrm{MAE}=1 / M_{1} M_{2} \sum_{i=1}^{M_{1}} \sum_{j=1}^{M_{2}}\left\|y(i, j)-y_{0}(i, j)\right\|_{L_{1}}
\end{gathered}
$$


where $\mathrm{MSE}=1 / M_{1} M_{2} \sum_{i=1}^{M_{1}} \sum_{j=1}^{M_{2}}\left\|y(i, j)-y_{0}(i, j)\right\|_{L_{2}}^{2}$ is the mean square error, $M_{1}, M_{2}$ are the image dimensions, $y(i, j)$ is the $3 \mathrm{D}$ vector value of the pixel $(i, j)$ of the filtered image, $y_{0}(i, j)$ is the corresponding pixel in the original uncorrupted image, and $\|\cdot\|_{L_{1}},\|\cdot\|_{L_{2}}$ are the $\mathrm{L}_{1}$ - and $\mathrm{L}_{2}$-vector norms, respectively;

$$
\operatorname{MCRE}=\sum_{i=1}^{M_{1}} \sum_{j=1}^{M_{2}}\left\|p_{i, j}-\hat{p}_{i, j}\right\|_{L_{2}}^{2} / M_{1} M_{2}
$$

where $p_{i, j}$ and $\hat{p}_{i, j}$ are the intersection points of $y(i, j)$ and $y_{0}(i, j)$ with the plane defined by the Maxwell triangle, respectively;

$$
\mathrm{NCD}=\sum_{i=1}^{M_{1}} \sum_{j=1}^{M_{2}}\left\|\Delta E_{L u v}(i, j)\right\|_{L_{2}} / \sum_{i=1}^{M_{1}} \sum_{j=1}^{M_{2}}\left\|E_{L u v}^{*}(i, j)\right\|_{L_{2}}
$$

where $\left\|\Delta E_{L u v}(i, j)\right\|_{L_{2}}=\left[\left(\Delta L^{*}(i, j)\right)^{2}+\left(\Delta u^{*}\right)^{2}+\left(\Delta v^{*}\right)^{2}\right]^{1 / 2}$ is the norm of color error; $\Delta L^{*}, \Delta u^{*}$, and $\Delta v^{*}$ are the difference in the $L^{*}, u^{*}$, and $v^{*}$ components, respectively, between the two color vectors that present the filtered image and uncorrupted original one for each a pixel $(i, j)$ of an image, and $\left\|E_{L u v}^{*}(i, j)\right\|_{L_{2}}=\left[\left(L^{*}\right)^{2}+\left(u^{*}\right)^{2}+\left(v^{*}\right)^{2}\right]^{1 / 2}$ is the norm or magnitude of the uncorrupted original image pixel vector in the $L^{*} u^{*} v^{*}$ space.

The 320x320 "Lena" color image was corrupted by $20 \%$ of impulsive noise. Table 1 shows that the performance criteria are often better for the proposed VMML filter in comparison when other filters in the most of cases.

Figure 1 exhibits the processed images for test image "Lena" explaining the impulsive noise suppression, and presenting the original image "Lena", image corrupted with noise probability occurrence of $20 \%$ for each color channel, and exhibiting the filtering results produced by the FASVM, VMMKNN, and the proposed VMML filter. From these results one can see that the proposed VMML filter has a better subjective quality in comparison with VMMKNN and FASVM filters.

We also use one frame of the 176x144 "Miss America" and "Flowers" video color sequences, which were corrupted by $15 \%$ and $10 \%$ of impulsive noise, respectively. Table 2 shows that the performance criteria in a frame of "Miss America" are often better for the proposed VMML filter in comparison when other filters in the most of cases.

Figure 2 exhibits the processed frames for test frame "Miss America" explaining the impulsive noise suppression according with Table 2. The restored frame with VMML filter has a better subjective quality in comparison with MAMNFE and VMMKNN filters. Figure 3 presents the subjective visual qualities of restored color frame "Flowers". The restored frame with VMML filter appears to have a similar subjective quality in comparison with FASVM and VMMKNN filters that have the better performance among the known color filters. 
Table 1. Comparative restoration results for $20 \%$ impulsive noise for color image "Lena"

\begin{tabular}{|l|c|c|c|c|}
\hline \multicolumn{1}{|c|}{ Algorithm } & PSNR & MAE & MCRE & NCD \\
\hline VM & 21.15 & 10.73 & 0.035 & 0.038 \\
\hline$\alpha-$ TM & 20.86 & 14.97 & 0.046 & 0.049 \\
\hline BVD & 20.41 & 12.72 & 0.043 & 0.045 \\
\hline GVD & 20.67 & 11.18 & 0.038 & 0.040 \\
\hline AGVD & 22.01 & 11.18 & 0.028 & 0.036 \\
\hline GVDF_DW & 22.59 & 10.09 & 0.028 & 0.039 \\
\hline MAMNFE & 22.67 & 9.64 & 0.027 & 0.035 \\
\hline VMMKNN (S) & 23.15 & 10.00 & 0.033 & 0.034 \\
\hline VMMKNN (A) & 23.07 & 10.01 & 0.033 & 0.035 \\
\hline FASVM & 24.80 & 5.00 & 0.025 & 0.017 \\
\hline VMML (S,E,ND) & 24.90 & 7.81 & 0.032 & 0.033 \\
\hline VMML (S,L,ND) & 25.81 & 6.49 & 0.026 & 0.016 \\
\hline VMML (S,U,ND) & 25.88 & 5.53 & 0.026 & 0.026 \\
\hline VMML (A,E,ND) & 22.65 & 12.32 & 0.034 & 0.040 \\
\hline VMML (A,L,ND) & 25.88 & 7.00 & 0.026 & 0.015 \\
\hline VMML (A,U,ND) & 26.52 & 5.36 & 0.022 & 0.015 \\
\hline VMML (S,E,D) & 26.13 & 3.36 & 0.024 & 0.027 \\
\hline VMML (S,L,D) & 26.46 & 2.90 & 0.023 & 0.027 \\
\hline VMML (S,U,D) & 26.47 & 2.79 & 0.023 & 0.025 \\
\hline VMML (A,E,D) & 25.25 & 4.48 & 0.030 & 0.023 \\
\hline VMML (A,L,D) & 26.59 & 3.00 & 0.022 & 0.029 \\
\hline VMML (A,U,D) & 26.73 & 2.74 & 0.021 & 0.025 \\
\hline
\end{tabular}

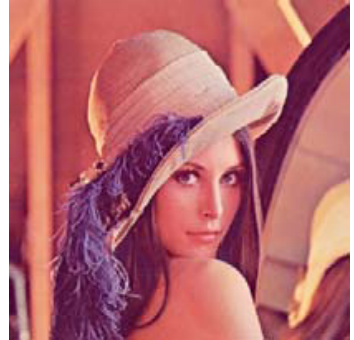

(a)

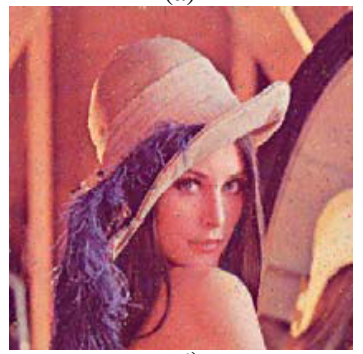

(d)

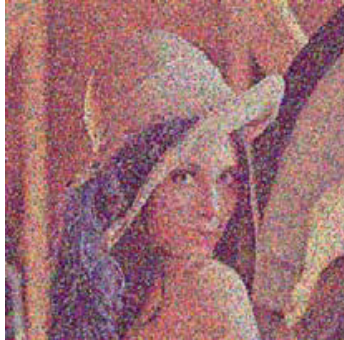

(b)

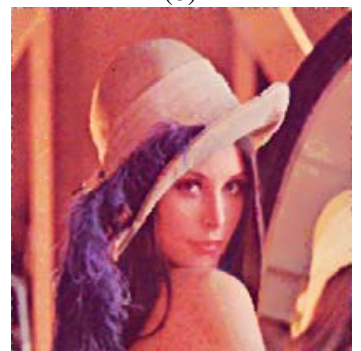

(e)

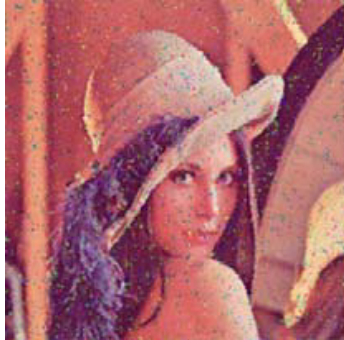

(c)

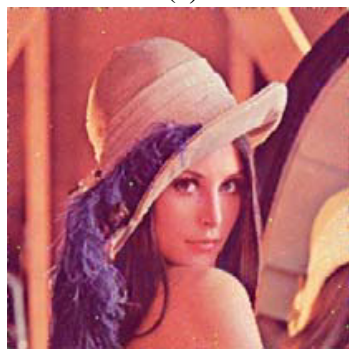

(f)

Fig. 1. Subjective visual qualities of restored color image "Lena", (a) Original test image "Lena", (b) Input noisy image (with $20 \%$ of impulsive noise), (c) VMMKNN filtering image, (d) FASVM filtered image (S), (e) Proposed VMML filtering image (S,E,ND), and (f) Proposed VMML filtering image (S,E,D) 
Table 2. Restoration results for $15 \%$ impulsive noise for a color frame of "Miss America"

\begin{tabular}{|l|c|c|c|c|}
\hline \multicolumn{1}{|c|}{ Algorithm } & PSNR & MAE & MCRE & NCD \\
\hline VM & 25.54 & 5.38 & 0.0371 & 0.0332 \\
\hline$\alpha$-TM & 24.47 & 6.54 & 0.0589 & 0.0251 \\
\hline BVD & 22.45 & 7.68 & 0.0379 & 0.0329 \\
\hline GVD & 23.56 & 9.12 & 0.0362 & 0.0308 \\
\hline AGVD & 26.97 & 5.24 & 0.0308 & 0.0302 \\
\hline GVDF_DW & 26.88 & 5.95 & 0.0311 & 0.0249 \\
\hline MAMNFE & 27.01 & 5.82 & 0.0390 & 0.0270 \\
\hline VMMKNN (S) & 28.20 & 3.86 & 0.0312 & 0.0140 \\
\hline VMMKNN (A) & 28.04 & 3.91 & 0.0317 & 0.0143 \\
\hline VMML (S,E,ND) & 27.87 & 4.41 & 0.0221 & 0.0189 \\
\hline VMML (S,L,ND) & 28.42 & 3.36 & 0.0218 & 0.0157 \\
\hline VMML (S,U,ND) & 28.56 & 2.62 & 0.0218 & 0.0150 \\
\hline VMML (A,E,ND) & 27.07 & 5.58 & 0.0225 & 0.0193 \\
\hline VMML (A,L,ND) & 28.44 & 3.27 & 0.0218 & 0.0157 \\
\hline VMML (A,U,ND) & 28.69 & 2.55 & 0.0217 & 0.0147 \\
\hline VMML (S,E,D) & 28.42 & 1.52 & 0.0218 & 0.0143 \\
\hline VMML (S,L,D) & 28.56 & 1.21 & 0.0218 & 0.0132 \\
\hline VMML (S,U,D) & 28.57 & 1.13 & 0.0217 & 0.0129 \\
\hline VMML (A,E,D) & 28.17 & 1.81 & 0.0219 & 0.0151 \\
\hline VMML (A,L,D) & 28.59 & 1.20 & 0.0217 & 0.0131 \\
\hline VMML (A,U,D) & 28.60 & 1.11 & 0.0217 & 0.0129 \\
\hline
\end{tabular}

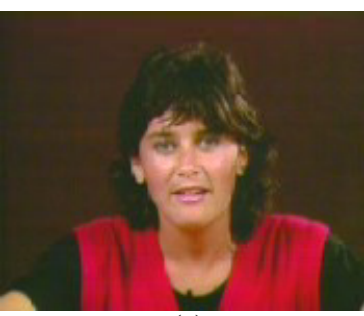

(a)

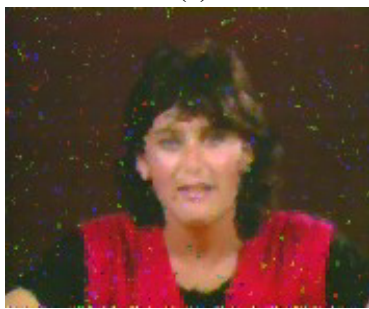

(d)

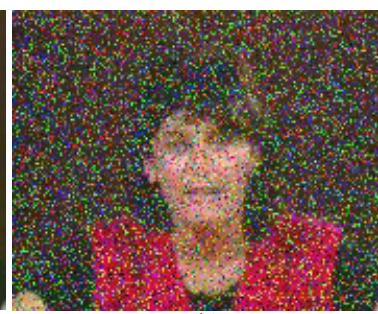

(b)

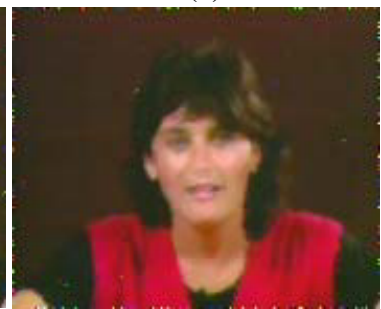

(e)

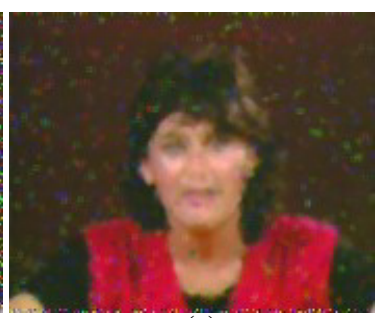

(c)

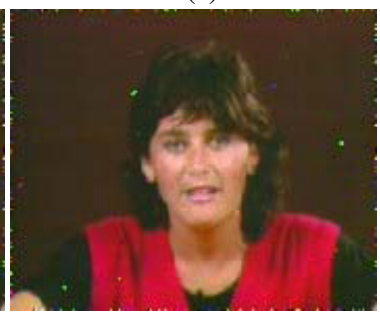

(f)

Fig. 2. Subjective visual qualities of restored color frame "Miss America", (a) Original test frame "Miss America", (b) Input noisy frame (with 15\% of impulsive noise), (c) MAMNFE filtered frame, (d) VMMKNN filtered frame (A), (e) Proposed VMML filtered frame (A,U,ND), and (f) Proposed VMML filtered frame (A,U,D) 


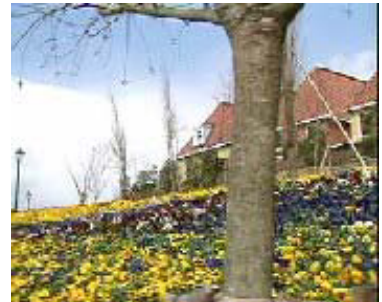

(a)

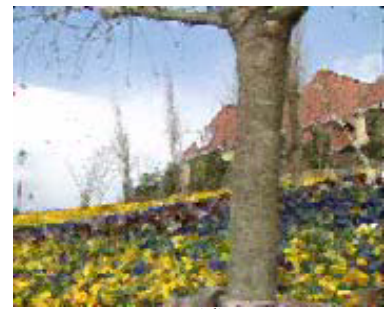

(d)

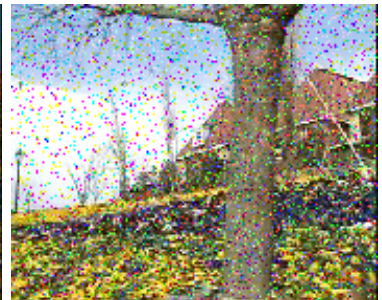

(b)

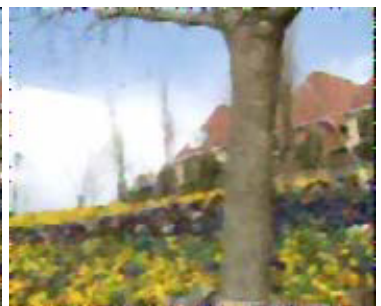

(e)

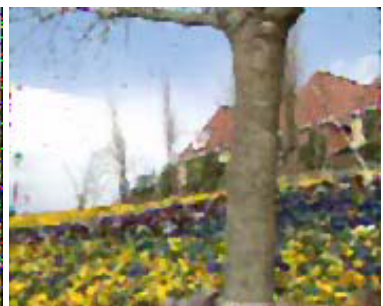

(c)

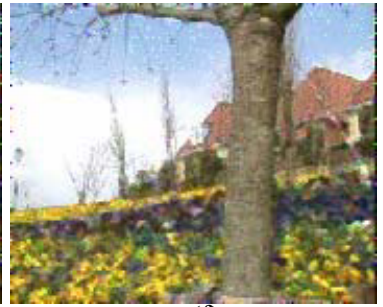

(f)

Fig. 3. Subjective visual qualities of restored color frame "Flowers", (a) Original test frame "Flowers", (b) Input noisy frame (with 10\% of impulsive noise), (c) VMMKNN filtered frame (S), (d) FASVM filtered frame, (e) Proposed VMML filtered frame (A,U,ND), and (f) Proposed VMML filtered frame (A,U,D)

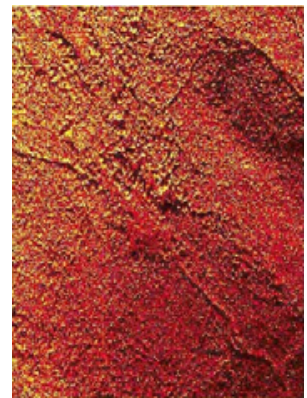

(a)

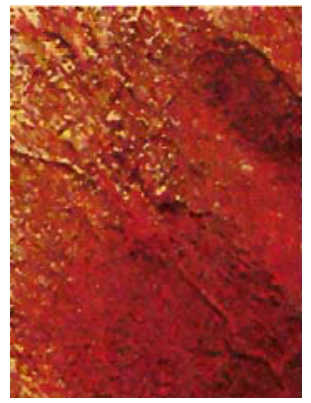

(b)

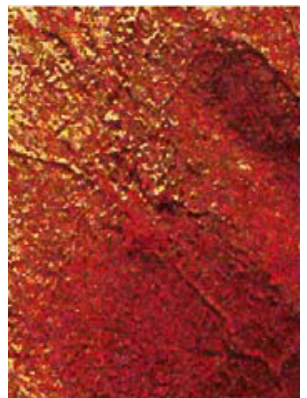

(c)

Fig. 4. Visual results of despeckled SAR image. a) Original image "Manzano", resolution 2m, source Sandia National Lab., b) Despeckled image with the proposed VMML filter (S,U,ND), c) Despeckled image with the proposed VMML filter (S,U,D).

From Tables 1-2 and Figures 1-3, one can see that the proposed VMML filter provides better noise suppression, detail preservation, and color retention when we use the impulsive noise detector in the most of cases, otherwise the MAE increase but the PSNR decrease. By other hand, the VMML filter has better performance when the impulsive noise percentage is high.

To demonstrate the performance of the proposed filtering scheme we applied it for filtering of $\mathrm{Ku}$ Band SAR images, which naturally have speckle noise. The filtering results are presented in Figure 4 for the image "Manzano" (forest near Manzano State 
Park, New Mexico). It is possible to see analyzing the filtering images that speckle noise can be efficiently suppressed, while the sharpness and fine feature are preserved using the proposed filter.

\section{Conclusions}

The proposed VMML filter is able to remove impulsive noise and preserve the edges and details in color imaging. The proposed filter uses the robust RM-estimator and utilizes an impulsive noise detector to provide better noise suppression, detail preservation, and color retention. The VMML filter has demonstrated better quality of image processing, both in visual and analytical sense in comparison with different known color image processing algorithms.

Acknowledgments. This work is supported by National Polytechnic Institute of Mexico.

\section{References}

1. Bovik, A.: Handbook of Image and Video Processing. Academic Press, San Diego (2000)

2. Plataniotis, K.N., Venetsanopoulos, A.N.: Color Image Processing and Applications. Springer, Berlin (2000)

3. Astola, J., Kuosmanen, P.: Fundamentals of Nonlinear Digital Filtering. CRC Press, Boca Raton (1997)

4. Plataniotis, K.N., Androutsos, D., Vinayagamoorthy, S., Venetsanopoulos, A.N.: Color image processing using adaptive multichannel filters. IEEE Trans. Image Process 6-7, 933-949 (1997)

5. Varela-Benitez, J.L., Gallegos-Funes, F.J., Ponomaryov, V.: RM L-filters for Real Time Imaging. In: IEEE 15th International Conference on Computing, pp. 43-48. IEEE Press, Mexico City (2006)

6. Varela-Benítez, J.L., Gallegos-Funes, F.J., Ponomaryov, V.I.: Real-time speckle and impulsive noise suppression in 3-D imaging based on robust linear combinations of order statistics. In: SPIE 6496 Real-Time Image Processing 2007, p. 64960H. SPIE Press, San Jose (2007)

7. Gallegos-Funes, F.J., Ponomaryov, V.I.: Real-time image filtering scheme based on robust estimators in presence of impulsive noise. Real Time Imaging 10(2), 69-80 (2004)

8. Pitas, I., Venetsanopoulos, A.N.: Nonlinear Digital Filters. Kluwer Academic Publishers, Boston (1990)

9. Aizenberg, I., Astola, J., Bregin, T., Butakoff, C., Egiazarian, K., Paily, D.: Detectors of the impulsive noise and new effective filters for the impulsive noise reduction. In: SPIE 5014 Image Processing, Algorithms and Systems II, pp. 419-428. SPIE Press (2003)

10. Trahanias, P.E., Karakos, D.G., Venetsanopoulos, A.N.: Directional processing of color images: Theory and experimental results. IEEE Trans. Image Process. 5, 868-880 (1996)

11. Ponomaryov, V., Gallegos-Funes, F., Rosales-Silva, A.: Real-Time Color Image Processing Using Order Statistics Filters. Journal of Mathematical Imaging and Vision 23(3), 315319 (2005)

12. Smolka, B., Lukac, R., Chydzinski, A., Plataniotis, K.N., Wojciechowski, W.: Fast adaptive similarity based impulsive noise reduction filter. Real-Time Imaging, 261-276 (2003) 